\title{
DECAY OF THE RED CU-S PAIR LUMINESCENCE IN GaP
}

K. Moser, R. Baumgartner, and w. Prettl

Institut für Angewandte Physik, Universität Regensburg, 8400 Regensburg, w.Germany

(Received 12 August, 1983 by E. Burstein)

The red Cu-S pair luminescence in GaP has been studied by time resolved spectroscopy at several photon energies between 1.39 and $1.77 \mathrm{eV}$ for times shorter than $40 \mathrm{\mu s}$. Superimposed on a long lasting luminescence a fast decay was observed showing the typical behaviour of donor-acceptor (DA) pair recombination. The transition probability $W_{m}$ was estimated to be $10^{6} \mathrm{~s}^{-1}$, being of the same order of magnitude as that of the other known DA pairs in GaP.

\section{Introduction}

Donor-acceptor (DA) radiative pair recombination has been extensively investigated for shallow impurities in GaP. Comparably little has been achieved, however, about DA pairs involving deep levels 1,2 . In part this is certainly due to the strong phonon coupling which prevents the observation of any electronic structure (zero phonon line) in the luminescence spectra ${ }^{3-5}$. Another complication of deep center recombination is the vast amount of nonradiative transitions ${ }^{6}$. The kinetics of shallow donor-Cu pair emission and the infrared quenching of luminescence have been previously investigated on a time scale up to several minutes ${ }^{7}$. In the present paper we report on the decay of $\mathrm{Cu}-\mathrm{S} D A$ Iuminescence for times shorter than $40 \mathrm{\mu s}$ and compare the results with the recombination of other donor-acceptor pairs like $\mathrm{Zn}-\mathrm{S}$ and $\mathrm{zn}-\mathrm{O}{ }^{2}$.

\section{Experimental}

The experiments were performed on commercially available Gap-crystals supplied by Wacker-Chemietronic. Measurements of the optical absorption and of the photoluminescence revealed the presence of the following impurities with approximate concentrations given in brackets: zinc $\left(<2 \cdot 10^{17} \mathrm{~cm}^{-3}\right)$, sulphur $\left(>10^{17} \mathrm{~cm}^{-3}\right)$, oxygen $\left(\sim 10^{17} \mathrm{~cm}^{-3}\right)$, and copper $\left(>10^{16} \mathrm{~cm}^{-3}\right)$. The samples were p-conducting having room temperature carrier concentrations not larger than $10^{17} \mathrm{~cm}^{-3}$. The crystals were immersed in liquid helium at $\mathrm{T}=1.6 \mathrm{~K}$. Interband transitions were excited at a quantum energy of $2.408 \mathrm{eV}$ by $3 \mathrm{~ns}$ long pulses of a dye laser. The power in the laser beam of $1 \mathrm{~mm}^{2}$ cross section was varied by calibrated filters up to $100 \mu \mathrm{J}$ per pulse. The photoluminescence was detected by a photomultiplier after passing through a double grating monochromator. The signals were stored and averaged over typically $10^{4}$ pulses by a transient recorder.

Besides these transient measurements the cw luminescence spectrum was determined by exciting with a $\mathrm{Kr}^{+}$-laser at $1.648 \mathrm{ev}$ and at
$2.336 \mathrm{eV}$ photon energies. As shown in Fig. 1 the spectrum excited at $2.336 \mathrm{ev}$ consists of a broad band with peak intensity at $1.65 \mathrm{eV}$ which is identified as emission due to $\mathrm{Cu}-\mathrm{S}$ pair re-

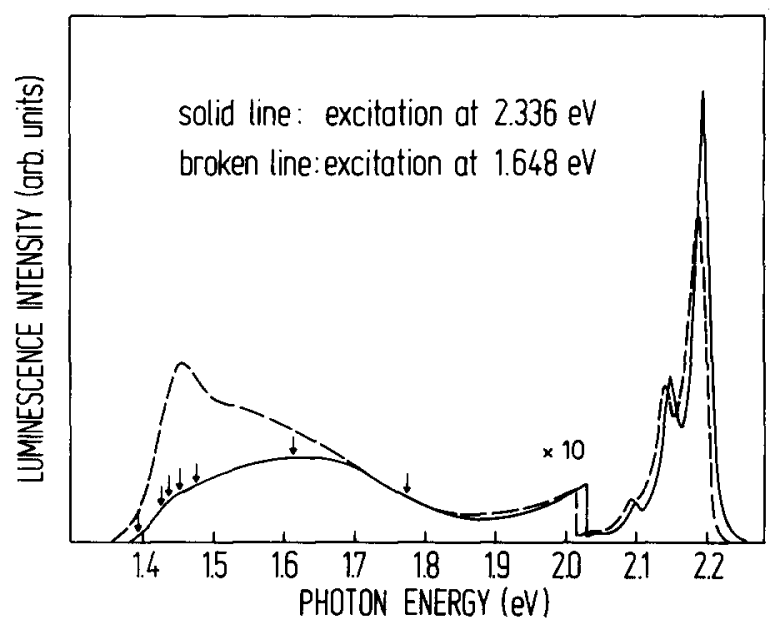

Fig. 1. Photoluminescence spectrum for $\mathrm{cw}$ excitation of the GaP crystal at $4.2 \mathrm{~K}$. The arrows indicate the detection quantum energies of the transient measurements. The structure around $2.2 \mathrm{eV}$ is caused by $\mathrm{zn}-\mathrm{S}$ DA pair decay (zero phonon line and phonon replicas). The energy shift of the peaks is due to different excitation powers.

combination in agreement with results of Monemar et al. 5,8. The $\mathrm{Cu}$ level involved in this transition is the so called "A-level" $(0.52 \mathrm{eV}$ above the valence band) whose physical nature is still not quite clear. On the low energy side a weak structure occurs which can be attributed to $\mathrm{z} n-\mathrm{O}$ pair recombinations ${ }^{2}$. This structure becomes much stronger compared to the $\mathrm{Cu}-\mathrm{S}$ emission, if an excitation photon energy of $1.648 \mathrm{eV}$ is applied (see Fig. 1) because the oxygen level involved is directly populated.

Results

The decay of the time resolved photolumi- 
nescence measured for various excitation pulse powers at different detection photon energies hud ranging from $1.393 \mathrm{ev}$ to $1.771 \mathrm{ev}$ is displayed in Figs. 2 and 3 on a logarithmic scale. The various detection quantum energies are in-

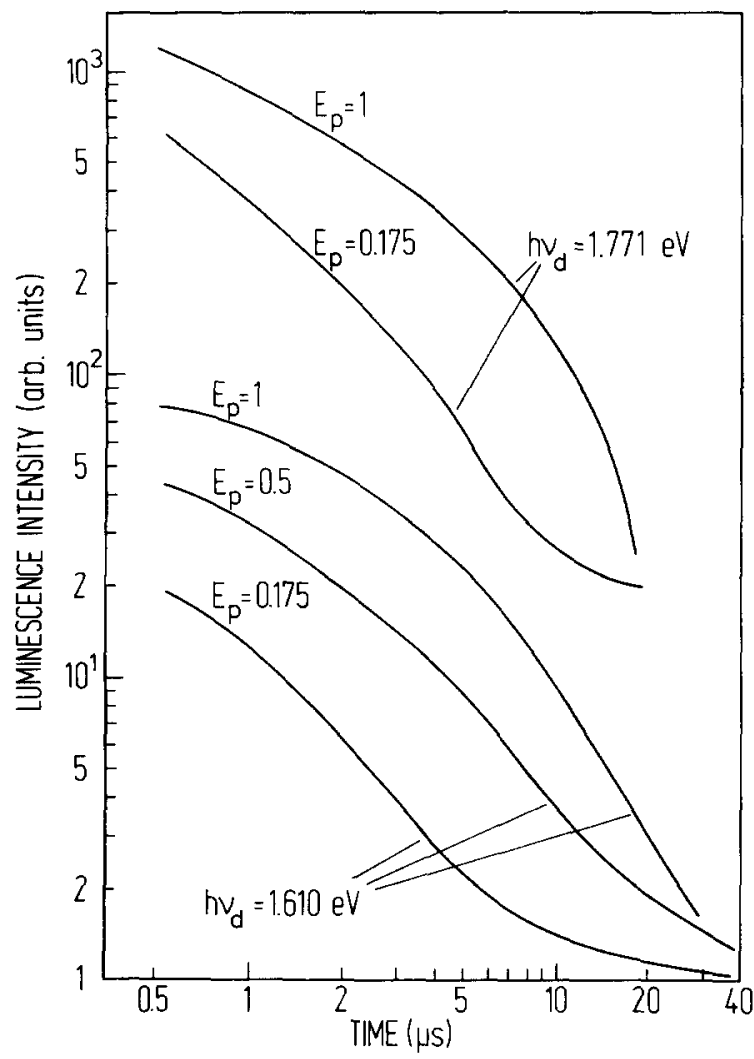

Fig. 2. Photoluminescence decay curves for detection quantum energies $h \nu_{d}=1.610$ and $1.771 \mathrm{eV}$ and different excitation pulse powers $\mathrm{E}_{\mathrm{p}}$ (arb. units). The vertical position is arbitrary.

dicated in Fig. 1 by arrows. The decay curves measured at the high energy side of the $\mathrm{Cu}-\mathrm{S}$ band $\left(\mathrm{h} v_{\mathrm{d}}=1.610 \mathrm{eV}\right.$ and $\left.1.771 \mathrm{eV}\right)$ are plotted in Fig. 2 and show that for decay times smaller than 5 to $10 \mu s$ after excitation the decay is faster when the exciting pulse power is lower. For longer times, however, a slower component becomes apparent which determines the luminescence after about $20 \mu \mathrm{s}$. The decay of the emission on the low energy side of the band is shown in Fig. 3. Again there are two characteristic transients, the slower one becoming more important after 2 to $7 \mathrm{\mu s}$. On reducing the excitation power the decay at $h v_{d}=1.476 \mathrm{ev}$ behaves similarly to that on the high energy side of the band shown in Fig. 2 .

\section{Discussion}

The experimental results show that an exponential fit is not appropriate to describe the observed time dependence of the luminescence. However, an exponential decay time may roughly be estimated for the fast decaying component yielding a time constant of about $10 \mu \mathrm{s}$. This is in order of magnitude agreement with the majority carrier lifetime measured by

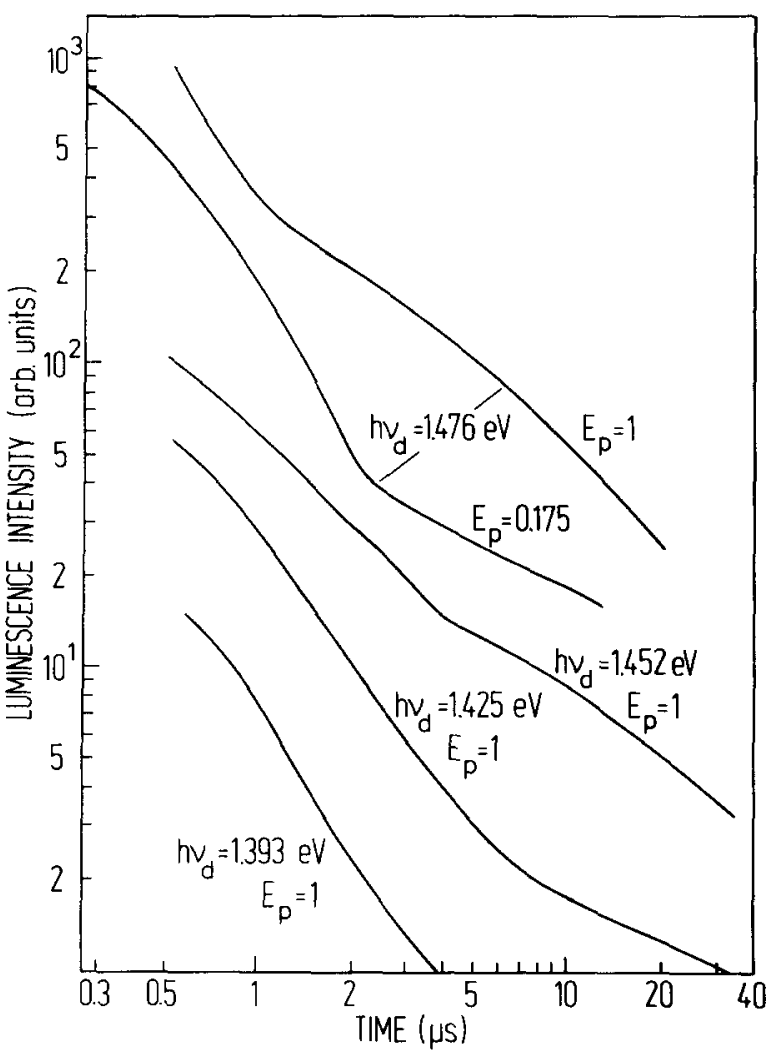

Fig. 3. The same as Fig. 2 but for $h v_{d}=1.393$, $1.425,1.452$ and $1.476 \mathrm{ev}$. The two different decay components are clearly visible.

photoconductivity in $\mathrm{GaP}: \mathrm{Cu}^{9}$ and the relaxation time of the interband excitation induced absorption which was attributed to a transient population of $\mathrm{Cu}$ levels 10 .

The decay curves at short times for photon energies at the high energy side of the lumines cence band, $h v_{d}=1.610$ and $1.771 \mathrm{ev}$, are similar to those of shallow DA pairs when donor and acceptor concentrations are very unequal and the impurity levels are saturated with charge carriers 1 . The time dependence can be described by a power law $t^{-n}$ with $n$ of the order of 1. The similarity to shallow impurity pair recombination must be due to the fact that the decay kinetics depends on the wave function of only one impurity, in this case the shallow Sdonors, and is independent of the unknown wave function of the deep cu-level. The decay curves behave in the same way for both photon energies because the luminescence consists at the lower quantum energy solely of phonon replicas as has been shown in ref.3-5. There are also at the higher photon energy strong phonon contributions so that the emission is most probably due to DA pairs of all distances. BY comparing the observed fast decay with experimental and theoretical results of $A . T$. Vink et al. ${ }^{2}$ we find the transition probability $W_{m}=W(R \rightarrow O)$ for the pair distance $\mathrm{R}$ extrapolated to zero to be about $10^{6} \mathrm{~s}^{-1}$. A more accurate determination of $W_{m}$ is not possible because only one decade of the fast component could be observed before 
the luminescence was dominated by the slow decay. The transition probability is of the same magnitude as that of other DA pairs in GaP ${ }^{2}$.

There is no clear explanation for the slowly decaying component of the emission. We can exclude the $\mathrm{z} n-\mathrm{O}$ complex, because its luminescence decays very fast and is not observable at $1.610 \mathrm{ev} 11$. This long time component may originate from very distant $\mathrm{Cu}-\mathrm{S}$ pairs, which decay very slowly. At low intensities this luminescence can be observed several minutes after the excitation 7 . This might indicate that the decay mechanism is somewhat different from that of other DA pairs. Other possible sources of the long time component could be the penetration of carriers into the bulk of the crystal, luminescence from a different impurity or interference from the slower $\mathrm{Zn}-\mathrm{S}$ pair decay, i.e. $\mathrm{Zn}-\mathrm{S}$ and $\mathrm{Cu}-\mathrm{S}$ pairs not being isolated. Also the observation that the decay becomes faster on reducing the exciting pulse laser energy cannot be definitely explained at present. This result is in contradiction to theory and other DA recombination measurements 1,2 . Again a larger penetration of carriers into the crystal at higher excitation energies might be a possible reason for this effect. on the other hand, negatively charged $\mathrm{Zn}$ acceptors, whose concentration is much larger than that of $\mathrm{Cu}$, could influence the $\mathrm{Cu}-\mathrm{S}$ recombination by the effect of stray coulomb fields.

Below about $h v_{d}=1.5 \mathrm{ev}$ the contribution of $\mathrm{zn}-\mathrm{O}$ pairs to the luminescence rises with decreasing photon energy and may even exceed that of the Cu-s pairs. Therefore we will not discuss these curves in detail. The transient behaviour, however, is similar to that at higher photon energies. Again the decay becomes faster when the exciting pulse energy is reduced and a long time component of unclear origin is present in the decay curves.

In conclusion we have measured for the first time the decay of the $\mathrm{Cu}-\mathrm{S}$ pair luminescence for short times. The experimental results show that with the exception of a slow decay component the decay kinetics behaves similarly to that of other identified DA pairs in GaP, even the decay probability is within one order of magnitude the same.

Financial support by the Deutsche Forschungsgemeinschaft is gratefully acknowledged.

\section{References}

1. D.G. Thomas, J.J. Hopfield, W.M. Augustyniak, Phys.Rev. 140, A202 (1965).

2. A.T. Vink, R.L.A. van der Heijden, A.C. van Amstel, J.Luminescence 9, 180 (1974).

3. B. Monemar, L. Samuelson, Phys.Rev. B 18 , 809 (1978).

4. L. Samuelson, B. Monemar, Phys.Rev. B 18, 830 (1978).

5. H.G. Grimmeiss, B. Monemar, L. Samuelson, Solid State Electr. 21, 1505 (1978).
6. C.H. Henry, D.V. Lang, Phys.Rev. B 15, 989 (1977).

7. H.G. Grimmeiss, B. Monemar, phys.stat.sol. (a) 19, 505 (1973).

8. B. Monemar, J.Luminescence 5, 472 (1972).

9. R.G. Schulze, P.E. Petersen, J.Appl.Phys. 45, 5307 (1974).

10. K. Moser, R. Baumgartner, W. Prettl, Solid State Commun. 45, 1051 (1983).

11. J.M. Dishman, I. Camlibel, Phys.Rev. B 6 . 1340 (1972). 
